\title{
Aspectos associados ao tabagismo e os efeitos sobre a saúde
}

\author{
Aspects associated with smoking and health effects \\ Aspectos asociados al tabaquismo y efectos sobre la salud
}

Thais Carolina Alves Cardoso

ORCID: https://orcid.org/0000-0002-0209-3840 Centro Universitário de Anápolis UniEVANGÉLICA, Brasil

E-mail: thaaiiscarolina@gmail.com

Adriano Ferro Rotondano Filho

ORCID: https://orcid.org/0000-0002-8176-6432 Centro Universitário de Anápolis UniEVANGÉLICA, Brasil E-mail: adrianoferro09@gmail.com

Laryssa Moura Dias

ORCID: https://orcid.org/0000-0002-3042-0124

Universidade Estadual de Campinas, Brasil E-mail: laryssamdias@hotmail.com

Jalsi Tacon Arruda

ORCID: https://orcid.org/0000-0001-7091-4850 Centro Universitário de Anápolis UniEVANGÉLICA, Brasil E-mail: jalsitacon@gmail.com

\begin{abstract}
Resumo
O hábito de fumar - tabagismo, está associado ao desenvolvimento de uma série de doenças. É algo comum desde os primórdios da pré-história da humanidade. O presente estudo realizou uma revisão integrativa da literatura para reunir aspectos associados ao tabagismo e os efeitos sobre a saúde. Foi realizada uma revisão integrativa de literatura a partir de estudos originais foram obtidos nas bases de dados SciELO, Google Acadêmico e LILACS. O termo "cigarro" referese ao cigarro feito de tabaco, embora seja utilizado para designar outros produtos semelhantes. O tabagismo já não é mais sinônimo apenas do consumo de cigarros convencionais feitos de tabaco. Outros produtos como o tabaco orgânico utilizado para fazer o charuto, o narguilé e o cigarro eletrônico disputam lugar com os maços. Todos os derivados do tabaco, que podem ser usados nas formas de inalação (cigarro, charuto, cachimbo, narguilé, cigarro de palha, eletrônico), aspiração (rapé) e mastigação (fumo-de-rolo), são nocivos à saúde. É muito importante monitorar a iniciação do tabagismo, principalmente entre os jovens, por essa ser uma ação passível de prevenção.
\end{abstract}

Palavras-chave: Cigarro; Fumo; Narguilé; Nicotina; Sistemas eletrônicos de liberação de nicotina.

\begin{abstract}
The habit of smoking - smoking, is associated with the development of a series of diseases. It has been common since the dawn of human prehistory. The present study carried out an integrative literature review to gather aspects associated with smoking and the effects on health. An integrative literature review was carried out based on original studies obtained from the SciELO, Google Scholar and LILACS databases. The term "cigarette" refers to a cigarette made from tobacco, although it is used to designate other similar products. Smoking is no longer synonymous only with the consumption of conventional cigarettes made from tobacco. Other products such as the organic tobacco used to make the cigar, the hookah and the electronic cigarette compete for place with the packs. All tobacco derivatives, which can be used in the forms of inhalation (cigarette, cigar, pipe, hookah, straw, electronic cigarette), aspiration (snuff) and chewing (roll smoke), are harmful to health. It is very important to monitor smoking initiation, especially among young people, as this is a preventable action.
\end{abstract}

Keywords: Cigarette; Smoke; Hookah; Nicotine; Electronic nicotine delivery systems.

\section{Resumen}

El hábito de fumar - fumar, está asociado al desarrollo de una serie de enfermedades. Ha sido común desde los albores de la prehistoria humana. El presente estudio realizó una revisión integradora de la literatura para recoger aspectos asociados al tabaquismo y sus efectos en la salud. Se realizó una revisión integradora de la literatura a partir de estudios originales obtenidos de las bases de datos SciELO, Google Scholar y LILACS. El término "cigarrillo" se refiere a un cigarrillo elaborado con tabaco, aunque se utiliza para designar otros productos similares. Fumar ya no es sinónimo únicamente del consumo de cigarrillos convencionales elaborados con tabaco. Otros productos como el tabaco orgánico que se usa para hacer el cigarro, el narguile y el cigarrillo electrónico compiten por el lugar con los paquetes. Todos los derivados del tabaco, que se pueden utilizar en forma de inhalación (cigarrillo, cigarro, pipa, narguile, pajita, cigarrillo 
electrónico), aspiración (rapé) y masticación (humo de rollo), son perjudiciales para la salud. Es muy importante vigilar el inicio del tabaquismo, especialmente entre los jóvenes, ya que se trata de una acción prevenible.

Palabras clave: Cigarrillo; Fumar; Narguile; Nicotina; Sistemas electrónicos de suministro de nicotina.

\section{Introduçãa}

Originário das Américas Central e Sul, o tabaco era muito utilizado em rituais religiosos como forma de aquisição de experiências transcendentais. Com as grandes navegações o fumo do tabaco foi disseminado pela Europa através dos marinheiros, soldados e até mesmo piratas. Sua importância econômica surgiu a partir do século XVII, sendo seu uso visto em todas as camadas da sociedade. Amplamente utilizado na forma de cigarro, mascado, rapé, cachimbo, charuto etc. (Nardi, 1985; Balbach, 1998).

O hábito de fumar é algo comum desde os primórdios da pré-história da humanidade. Uma epidemia no consumo do tabaco teve início no final do século XIX, impulsionado pela invenção da máquina de fabricar cigarros. Já no século XX passou a ser influenciado pela indústria da propaganda, o cinema, as grandes guerras e a ampliação da circulação de mercadorias e de pessoas (Bernardes-Souza et al., 2018).

De acordo com a avaliação da Organização Mundial de Saúde, na década de 1990-1999, um total de 3 a 4 milhões de pessoas morreram anualmente no mundo de doenças induzidas pelo tabaco. Em outra abordagem relacionada ao tema, o Programa Nacional de Controle do Tabaco do Ministério da Saúde Pública destacou que se trata de uma situação que afeta fundamentalmente os países em desenvolvimento, e que somente no ano 2000 4,8 milhões de pessoas morreram por doenças relacionadas a esse hábito, sendo 3,8 milhões de homens e 1 milhão de mulheres. Em 2025 esse número pode aumentar para 10 milhões de indivíduos (Santos, 2018).

O tabagismo já não é mais sinônimo apenas do consumo de cigarros. Outros produtos como o tabaco orgânico (ou charuto), o narguilé e o cigarro eletrônico tomam o lugar dos maços vendidos em padarias e bancas de jornal. Todos os derivados do tabaco, que podem ser usados nas formas de inalação (cigarro, charuto, cachimbo, narguilé, cigarro de palha, eletrônico), aspiração (rapé) e mastigação (fumo-de-rolo), são nocivos à saúde (Urrutia-Pereira et al., 2019).

O fumo é importante causa de perda de saúde. Além disso, está associado ao desenvolvimento de uma série de doenças respiratórias, cardiovasculares e neoplásicas. Estima-se que a mortalidade geral seja duas vezes maior nos fumantes se comparado aos não-fumantes. Ainda assim, apesar de ser um importante fator de risco e, cuja remoção repercute significativamente na redução da morbimortalidade, os dados associados a prevalência de tabagismo na população brasileira são esparsos. As estimativas oscilam entre $32 \%$ e $42 \%$ da população, sendo maioria homens entre a $3^{\circ}$ e $6^{\circ}$ década de vida e posição socioeconômica inferior (Moreira et al., 1995).

A indústria do tabaco entende que os jovens, referidos como "aprendizes", são o mercado mais importante. Atualmente no século XXI, o fumo e o álcool são as substâncias mais consumidas pelos jovens, o que preocupa as instituições de saúde (Silva et al., 2020). São consideradas drogas portáteis para o consumo de outras como: maconha, cocaína, pasta base, estimulantes, sedativos, dentre outras. Além disso, $90 \%$ dos fumantes iniciaram o hábito antes dos 19 anos, e foram detectados problemas em crianças e adolescentes fumantes (como distúrbios de comportamento agressivo e tímido) (Gorrita Pérez et al., 2012). Entre os fatores que podem predispor ou, ao contrário, ser protetores quanto ao consumo de tabaco, cita-se o ambiente familiar e sociocultural em que vivem os adolescentes. Diante dessas informações o presente estudo realizou uma revisão integrativa da literatura para reunir aspectos associados ao tabagismo e os efeitos sobre a saúde.

\section{Metodologia}

Trata-se de uma revisão integrativa de literatura que permite a síntese de diversos estudos publicados, possibilitando 
inferir conclusões a respeito da temática (Pereira et al., 2018). Foi realizada a partir de 23 artigos, na língua portuguesa, espanhola e inglesa, sem restrição do ano de publicação. Estes estudos originais foram obtidos nas bases de dados Scientific Electronic Library Online (SciELO), Google Acadêmico e Literatura Latino-Americana e do Caribe em Ciências da Saúde (LILACS). Foram utilizados os seguintes descritores em Ciências da Saúde (DeCS): "hábito de fumar" e "hábito de fumar maconha", em português, inglês ou espanhol, sem restrição de ano de publicação. Os critérios de inclusão dos estudos foram: artigos originais completos e disponíveis na íntegra; revisões e relatos de casos. Foram desconsiderados os artigos que não se enquadraram nesses critérios, outros tipos como livros, monografia, dissertações, teses, comentários e cartas ao leitor/editor. Além disso, foram excluídos trabalhos que não apresentavam correlação com o tema. O artigo selecionado foi lido na íntegra para determinar sua elegibilidade para posterior inclusão no estudo. Caso contrário, essas produções foram excluídas.

\section{Resultados e Discussão}

Partindo das leituras seletivas para a construção do presente estudo, cinco categorias foram individualizadas para discussão da temática, sendo: histórico do fumo, tipos, epidemiologia, morbimortalidade e hábito de fumar outras substâncias, que foram abordadas a seguir.

\subsection{Histórico}

O tabaco é uma planta cujo nome científico é Nicotiana tabacum, da qual é extraída a substância nicotina. Seu uso surgiu aproximadamente no ano 1.000 a.C., nas sociedades indígenas da América Central, nas proximidades da cidade de Tobaco, região de Yucatán. Relatos de colonizadores espanhóis em 1520, avistaram pela primeira vez a planta sendo usada pelos índios. Também existem relatos de que a origem do nome da planta tabaco foi denominada pelos colonizadores em homenagem à localidade onde a encontraram pela primeira vez (Balbach, 1998).

No Brasil, no início do século XVI, cultivava tabaco em quase todas as tribos indígenas, desde que os primeiros portugueses desembarcaram no País. Para os índios brasileiros, essa planta possuía caráter sagrado e origem mítica, seu uso era, geralmente, limitado a ritos mágico-religiosos, para evocar os deuses e predições, bem como para fins medicinais, para enxaquecas, cura de ferimentos e até mesmo dores de estômago, e seu uso era reservado exclusivamente aos pajés (Nardi, 1985; Balbach, 1998). O tabaco era consumido de diferentes maneiras entre os indígenas podendo ser comido, bebido, mascado, aspirado e fumado, entretanto o hábito de fumar predominava e esta forma de consumo acabou se difundindo pelo mundo. De planta mágico-religiosa, o tabaco passou a ser um produto comercial das colônias europeias, e mais particularmente, das Antilhas, da Virgínia (a partir de 1612) e do Brasil (Nardi, 1985).

Assim, rapidamente o cultivo e comércio de tabaco no Brasil colonial passou a ter importância destacada, a ponto de já no decorrer do século XVII o seu comércio ter várias legislações e taxações, passando a figurar entre os principais produtos exportados durante o período Imperial. Esta importância está marcada até os dias atuais no brasão das Armas da República, onde o tabaco e o ramo de café constituem o coroamento deste símbolo da nacionalidade brasileira (Nardi, 1985; Balbach, 1998).

\subsection{Tipos}

O termo "cigarro" refere-se ao cigarro feito de tabaco, embora seja utilizado para designar outros produtos semelhantes, como cigarro de Cannabis sativa (maconha). Um cigarro é diferente de um charuto ou cigarrilha. O cigarro tradicional, aquele encontrado em carteiras em diversos lugares de fácil acesso, é constituído por folhas de tabaco, de corte fino enroladas numa mortalha de papel que pode ser fumado. $\mathrm{O}$ tabaco orgânico, consumido como charuto, talvez seja o mais semelhante ao cigarro. Os charutos são constituídos somente por folhas de tabaco integral. No entanto, não contém filtro - a não ser que se compre 
separadamente. Por isso, pode ser considerado mais prejudicial que o cigarro (Soares, 2018; INCA, 2020).

O narguilé é uma espécie de cachimbo utilizado por indígenas da Ásia para fumar tabaco e outras substâncias, como flores, temperos, frutas, café, maconha ou haxixe. As rotas de comércio pela Índia e China disseminaram o narguilé no Oriente (Maziak et al., 2015). Além do termo "Narguilé", de origem persa, há variantes como arguile, muito usada em países árabes, também chamado de shisha especialmente na África e em outros países de língua árabe, ou ainda hookah na Índia ou em outros países de língua inglesa, entre outros nomes (Menezes et al., 2015).

Narguilé se trata de uma espécie de cachimbo d'água utilizado para fumar tabaco aromatizado e, ocasionalmente, maconha ou ópio. Há diferenças regionais no formato e funcionamento, é um dispositivo no qual uma mistura de tabaco é aquecida e a fumaça gerada passa por um filtro de água antes de ser aspirada pelo fumante, por meio de uma longa mangueira e um bocal (Malta et al., 2018). Por utilizar mecanismos de filtro, o consumo de narguilé é visto por alguns como menos nocivo à saúde. No entanto, vários estudos revelaram que é mais prejudicial do que o cigarro convencional (Souza et al., 2018; WHO, 2020).

Popular entre os mais jovens, é muito comum o uso de aromatizantes e flavorizantes ao tabaco, que torna o produto mais palatável, fornecendo um sabor agradável durante as sessões de fumo. Essa é uma estratégia da indústria do tabaco para atrair mais jovens, colaborando com a disseminação desse dispositivo de fumar, pois o cheiro/sabor pode atrair novos usuários e reforçar o comportamento de fumar para o fumante usual. Além disso, o narguilé possui uma característica peculiar: um único cachimbo é compartilhado por várias pessoas simultaneamente. Tal fato reforça a socialização do cachimbo, algo muito atraente, especialmente para os jovens que buscam "enturmar" (Maziak et al., 2015).

Cerca de 100 milhões de pessoas no mundo usam o tabaco e o uso de narguilé, após 45 minutos de sessão, eleva as concentrações plasmáticas de nicotina, de monóxido de carbono expirado e os batimentos cardíacos, equivale a tragar 100 cigarros (Menezes et al., 2015). Ademais, maior exposição a metais pesados, altamente tóxicos e de difícil eliminação, como o cádmio. Nesse caso, o culpado não é apenas o tabaco e sua composição específica para este produto, mas também as essências e o carvão, utilizado para aquecer a mistura. Todavia, os riscos do uso do narguilé não estão relacionados somente ao tabaco, mas também a doenças infectocontagiosas: o hábito de compartilhar o bucal entre os usuários pode resultar na transmissão de doenças como herpes, hepatite $\mathrm{C}$ e tuberculose (Malta et al., 2018). É importante destacar que a dependência pode ser a porta de entrada para o uso regular de outros produtos de tabaco, e a adição de maconha durante as sessões também torna-se um hábito frequente entre os usuários, resultando em maiores danos à saúde (Souza et al., 2018).

O cigarro eletrônico (CE), também chamado de vape, e-cigarro, e-ciggy ou e-cigarette, é um dispositivo eletrônico para fumar. É um aparelho mecânico-eletrônico alimentado por bateria, cujo no interior há um espaço para a inserção do cartucho ou refil de nicotina líquida, disponível nas concentrações que variam entre zero e $36 \mathrm{mg} / \mathrm{mL}$ ou mais em alguns casos (Carvalho, 2018). O atomizador aquece e vaporiza a nicotina. Durante a sucção da tragada, o sensor é acionado e deflagra a ação do microprocessador responsável por ativar tanto a bateria quanto à luz de LED (pequeno dispositivo luminoso), geralmente de cor laranja, localizado na ponta do cigarro que simula a brasa durante a tragada (Knorst et al., 2014).

Algumas marcas dizem comercializar aparelhos com ou sem nicotina. Ao tragar os vapers (denominação das pessoas que utilizam esse aparelho) absorvem os vapores gerados a partir de soluções conhecidas como e-liquids ou $e$-juices que contêm solventes e várias concentrações de nicotina, água e aromatizantes. Os solventes mais populares usados são a glicerina (geralmente de origem vegetal) e o propilenoglicol, além do glicerol que pode estar presente ou não. Os e-liquids não apresentam alcatrão, monóxido de carbono e nenhuma das outras substâncias comumente encontradas em produtos do tabaco (UrrutiaPereira et al., 2019).

Esse tipo de dispositivo para fumo está cada vez mais popular no Brasil e no mundo. Tal e qual o narguilé os vapers causam danos à saúde do fumante. Vários casos de $e$-liquids falsificados ocasionaram mortes em 6 jovens usuários nos EUA em 
2019, de uma "Doença Pulmonar" ainda pouco conhecida. No Brasil, esse dispositivo é proibido e tal proibição é regulada pela Agência Nacional de Vigilância Sanitária (ANVISA) (Urrutia-Pereira et al., 2019). Apesar disso, os reais efeitos destes produtos sobre a saúde dos usuários ainda não são totalmente conhecidos, mesmo que os recentes casos de mortes e doenças respiratórias nos EUA e no Brasil associadas a eles tenham ajudado a levantar suspeitas contra os cigarros eletrônicos.

Dentre os problemas observados no uso o fato de que o glicol e o vapor de glicerol, que são componentes da maioria dos cigarros eletrônicos, atuam como irritantes das vias aéreas superiores, além de que o contato com o vapor de glicol pode secar mucosas e olhos. Quanto aos efeitos fisiológicos do CE verificou-se irritação da faringe e tosse seca com o uso inicial do produto, além de níveis de cotinina plasmática e comprometimento da função respiratória semelhante ao cigarro tradicional (Nogueira, 2014).

A prevalência de conhecimento do CE em um estudo observacional, de delineamento transversal, feito com alunos de graduação da Universidade Federal de Mato Grosso (Campus Cuiabá), em 2015, foi de 37\%, e houve uma diferença estatisticamente significante entre os sexos, sendo que 59\% eram do sexo masculino e $41 \%$ eram do sexo feminino. Houve associação linear e inversamente proporcional em relação à idade do estudante, ou seja, quanto mais jovem é o estudante, maior é a chance de conhecer o dispositivo eletrônico. Além disso, o conhecimento do $\mathrm{CE}$ associou-se à maior nível de escolaridade dos pais, visto que, em 40,4\%, o pai possuía nível superior e, em 47,8\%, a mãe possuía tal graduação (Oliveira et al., 2018).

Uma revisão sobre as propagandas de cigarros eletrônicos em vários sites em 2011, esses constatavam que 95\% dos anúncios alegavam, de maneira explícita ou implícita, que o produto era mais saudável; 93\% que era mais barato; 95\% afirmavam ser mais limpo quando comparado aos cigarros regulares; $88 \%$ propagavam o uso em qualquer lugar; $71 \%$ fomentavam o uso para contornar as políticas de controle do tabagismo, pois afirmavam que não produziam o fumo passivo (76\%); $73 \%$ inferiam que são modernos com apelo aos jovens; $64 \%$ tinham relação com a cessação do fumo e $22 \%$ utilizaram a figura do médico. Doces, frutas, café e uma infinidade de sabores estavam presentes na maioria dos sites; $44 \%$ faziam alusão ao aumento do status social; $32 \%$ mencionavam a melhora da atividade social; $31 \%$ convidavam ao romance e $22 \%$ propagavam o uso por celebridades (Carvalho, 2018).

Tal popularização pode ter ocorrido em consequência da maior disseminação por parte das companhias produtoras de cigarros eletrônicos e propagandas para estimular seu uso. Os principais argumentos usados pela indústria são os benefícios à saúde em comparação ao cigarro tradicional de tabaco e a minimização da exposição passiva (Jeon et al., 2016).

\subsection{Epidemiologia}

Existem mais de um bilhão de fumantes no mundo e $80 \%$ deles vivem em 24 países, sendo dois terços em países de baixa e média renda onde a carga das doenças e mortes tabaco relacionadas é mais pesada. Estima-se que os fumantes atuais consumam cerca de seis trilhões de cigarros todos os anos (WHO, 2020).

No Brasil, em 2013, a prevalência do uso de tabaco em pessoas na idade de 18 anos ou mais foi estimada em 15\%, sendo 19,2\% entre homens e 11,2\% entre mulheres, essa prevalência correspondente a 21,8 milhões de brasileiros. A prevalência foi menor entre os jovens de 18 a 24 anos (10,7\%) e maior entre os adultos de 40 a 59 anos (19,4\%). Quanto ao nível de instrução, a menor prevalência de adultos usuários do tabaco foi encontrada entre aqueles com nível superior $(8,8 \%)$, e a maior nos indivíduos sem instrução ou com Ensino Fundamental incompleto (20,2\%) (Malta, 2015).

Em relação as características de cor da pele ou etnia, foi possível observar que entre os brancos entrevistados 13,1\% declaram-se usuários atuais do tabaco, seguidos de $16,4 \%$ de pardos e 17,8\% de pretos. A prevalência do fumo de tabaco foi maior na área rural, comparativamente a área urbana do país. Segundo Unidades da Federação, as maiores prevalências foram observadas nos estados do Acre, Mato Grosso do Sul, Paraná e Minas Gerais. A prevalência do fumo do tabaco, em 2015, foi de $14,7 \%$, sendo para o sexo masculino de 18,9\% e 11\% feminino (Malta, 2018). 
Considera-se o tabagismo um dos principais fatores de risco associados a doenças crônicas não transmissíveis e, além disso, é a principal causa mundial prevenível de morbidade e mortalidade, sendo responsável por cerca de 6 milhões de mortes ao ano. Estima-se, a partir das evidências epidemiológicas disponíveis, que o tabagismo esteja relacionado a cerca de 50 doenças. Destacando-se doenças cardiovasculares, respiratórias e neoplásicas. Além disso, foi demonstrado que 45\% dos óbitos por doença coronariana aguda (infarto agudo do miocárdio - IAM), 85\% por doença pulmonar obstrutiva crônica (DPOC), 25\% por doenças cerebrovasculares e 30\% por cânceres podem ser atribuídos ao consumo de derivados do tabaco (Pinto et al., 2015).

No período de consumo desses produtos são introduzidas no organismo cerca de 4.720 substâncias tóxicas. A principal substância química psicoativa que está presente é a nicotina, extremamente viciante (Santos, 2018). Além disso, o monóxido de carbono - o mesmo gás venenoso que sai do escapamento de automóveis; e o alcatrão constituído por aproximadamente 60 substâncias cancerígenas, como agrotóxicos e elementos radioativos. O fumo produzido pelos cigarros é um aerossol com mais de 4000 compostos químicos diferentes (Ickes et al., 2020).

Outrossim, o usuário de produtos de tabaco é exposto continuamente a mais de 4 mil substâncias tóxicas, muitas delas cancerígenas. Logo, essa exposição faz do tabagismo o mais importante fator de risco isolado de doenças graves e fatais. Entre elas, estima-se que os fumantes corram riscos muito mais elevados de adoecerem por cânceres e outras doenças crônicas comparado aos não-fumantes. Sendo, o pulmão, laringe, pâncreas, fígado bexiga e rim os principais órgãos acometidos por doenças neoplásicas associados ao consumo do tabaco. Ademais, as pesquisas apontaram que até mesmo filhos de pais fumantes, quando comparados aos filhos de não-fumantes, apresentam maior frequência de infecções e outros problemas respiratórios (INCA, 2020).

\subsection{Hábito de fumar outras substâncias}

Em relação ao uso do tabaco entre jovens e os fatores associados, destacam-se a exposição domiciliar ao cigarro, no ambiente familiar, na escola e a influência dos amigos. A da sociedade moderna exige que o jovem se inclua no mundo high tech, com forte apelo consumista por marcas e produtos, globalizando costumes e tendências. O jovem universitário também é exposto a essas "obrigações" da vida acadêmica e se vê diante do dilema: me encaixo na turma ou não? Embora a propaganda de cigarros esteja proibida nos meios de comunicação, está sempre presente nas mídias, jogos eletrônicos, seriados e filmes. A indústria promove propagandas e outras estratégias de marketing que associam o ato de fumar ao rito de passagem para o mundo adulto e o cigarro como um ícone ideal de autoimagem, incentivando a experimentação e a iniciação ao consumo de drogas (lícitas ou ilícitas) (Garcia et al., 2020).

A maconha é a droga ilícita mais usada em todo o mundo, sendo geralmente intermitente e limitado esse uso. No entanto, estima-se que até $10 \%$ dos que experimentaram maconha se tornam usuários diários e $20 \%$ a $30 \%$ desses a consomem semanalmente (Jungerman et al., 2005). Devido seus efeitos nocivos não serem tão óbvios, alguns autores sugerem que dar-seá mais atenção a outras drogas comparado a maconha. Entretanto, tornou-se extremamente importante sua investigação por profissionais da saúde devido aos prejuízos associados ao uso da maconha implicados em transtornos mentais. Sabe-se que a maconha produz uma série de alterações cognitivas cursando com déficits em várias áreas, incluindo aprendizado verbal, memória de curto prazo, atenção e funções executivas (Garcia et al., 2020).

Outrossim, os usuários podem apresentar ainda euforia leve, alterações da percepção, excitação, dissociação de ideias, aumento da sensibilidade auditiva, impulsos irresistíveis, ilusões e alucinações. Em relação a efeitos físicos, observa-se desequilíbrio da capacidade de localização, aumento do ritmo cardíaco, queda da pressão arterial, hiperemia conjuntival e alívio das náuseas (Garcia et al., 2020).

Observou-se que a maconha possui cerca de 400 compostos químicos, contando com 60 canabinoides, considerados princípios ativos específicos. Entre eles destacam-se o tetrahidrocanabinol (THC) e o canabidiol (CDB) por suas propriedades 
medicinais, tais como ação analgésica, antitumoral, aumento de apetite, relaxamento muscular, redução da insônia e melhora do humor. Entretanto, sabe que o THC, apesar de seus efeitos terapêuticos é também a principal substância psicoativa da planta, logo, existe um potencial associado a dependência química (Garcia et al., 2020).

\section{Conclusão}

Os resultados evidenciam as diversas formas de fumar, todas elas prejudiciais à saúde. Todos os estudos científicos apontam efeitos danosos desse hábito sempre associado ao desenvolvimento de câncer de pulmão, câncer de boca, bexiga e leucemia; doenças respiratórias, coronarianas, doença periodontal além de outras comorbidades. Por isso, é importante monitorar a iniciação do tabagismo entre os jovens, por essa ser uma ação passível de prevenção. Estimular a prevenção e a iniciação ao consumo de drogas (lícitas ou ilícitas) é fundamental para a promoção de ações inclusivas, educativas e de orientação preventiva quanto aos comportamentos de risco, aos quais são mais suscetíveis entre os jovens.

\section{Referências}

Balbach, A. (1998). O fumo e a saúde. Veneno lento. (22a ed.), Editora Edificação do lar.

Bernardes-Souza, B., Patruz Ananias De Assis Pires, F., Madeira, G. M., Felício Da Cunha Rodrigues, T., Gatzka, M., Heppt, M. V., Omlor, A. J., Enk, A. H., Groneberg, D. A., Seeger, W., von Kalle, C., Berking, C., Corrêa, P., Suhre, J. L., Alfitian, J., Assis, A., \& Brinker, T. J. (2018). Facial-Aging Mobile Apps for Smoking Prevention in Secondary Schools in Brazil: Appearance-Focused Interventional Study. JMIR public health and surveillance, 4(3), e10234. https://.org/10.2196/10234

Carvalho, A. de M. (2018). Cigarros Eletrônicos: o que sabemos? Estudo sobre a composição do vapor e danos à saúde, o papel na redução de danos e no tratamento da dependência de nicotina. Revista Brasileira de Cancerologia, 64(4), 587-9.

Garcia, T. R., Cruz, M. C. A., Silva, G. de O. A., Cardoso, E. F., \& Arruda, J. T. (2020). Canabidiol para o tratamento de pacientes com Síndrome de West e epilepsia. Research, Society and Development, 9(9), e420997267. 10.33448/rsd-v9i9.7267.

Gorrita Pérez, R. R., Gilvonio Cárdenas, A., \& Hernández Martínez, Y. (2012). Caracterización del hábito de fumar en un grupo de escolares adolescentes. Revista Cubana de Pediatría, 84(3), 256-264.

Ickes, M. J., Wiggins, A. T., Rayens, M. K., \& Hahn, E. J. (2020). Student Tobacco Use Behaviors on College Campuses by Strength of Tobacco Campus Policies. American journal of health promotion: AJHP, 34(7), 747-753. https://.org/10.1177/0890117120904015

INCA. Instituto Nacional de Câncer José Alencar Gomes da Silva. (2020). Observatório da Política Nacional de Controle do Tabaco. http://www.inca.gov.br/observatorio-da-politica-nacional-de-controle-do-tabaco/convencao-quadro.

Jeon, C., Jung, K. J., Kimm, H., Lee, S., Barrington-Trimis, J. L., McConnell, R., Samet, J. M., \& Jee, S. H. (2016). E-cigarettes, conventional cigarettes, and dual use in Korean adolescents and university students: Prevalence and risk factors. Drug and alcohol dependence, 168, 99-103. https://.org/10.1016/j.drugalcdep.2016.08.636

Jungerman, Flavia S, Laranjeira, Ronaldo, \& Bressan, Rodrigo A. (2005). Maconha: qual a amplitude de seus prejuízos? Brazilian Journal of Psychiatry, 27(1), 5-6. https://dx..org/10.1590/S1516-44462005000100003

Knorst, M. M., Benedetto, I. G., Hoffmeister, M. Costa, \& Gazzana, M. B. (2014). Cigarro eletrônico: o novo cigarro do século 21? Jornal Brasileiro de Pneumologia, 40(5), 564-572. https://dx..org/10.1590/S1806-37132014000500013

Malta, D. C., Hallal, A. L. C., Machado, Í. E., Prado, R. R. do, Oliveira, P. P. V. de, Campos, M. O., \& Souza, M. de F. M. de. (2018). Fatores associados ao uso de narguilé e outros produtos do tabaco entre escolares, Brasil, 2015. Revista Brasileira de Epidemiologia, 21(1), e180006. https://.org/10.1590/1980549720180006.supl.1

Malta, D. C., et al. (2015). Uso e exposição à fumaça do tabaco no Brasil: resultados da Pesquisa Nacional de Saúde 2013. Epidemiologia e Serviços de Saúde, 24(2), 239-248. https://.org/10.5123/S1679-49742015000200006

Maziak, W., Taleb, Z. B., Bahelah, R., Islam, F., Jaber, R., Auf, R., \& Salloum, R. G. (2015). The global epidemiology of waterpipe smoking. Tobacco control, 24 Suppl 1(Suppl 1), i3-i12. https://.org/10.1136/tobaccocontrol-2014-051903

Menezes, A. M. B., et al (2015). Frequência do uso de narguilé em adultos e sua distribuição conforme características sociodemográficas, moradia urbana ou rural e unidades federativas: Pesquisa Nacional de Saúde (PNS), 2013. Revista Brasileira de Epidemiologia, 18(Suppl. 2), 57-67. https://.org/10.1590/19805497201500060006

Moreira, L. B., Fuchs, F. D., Moraes, R. S., Bredemeir, M., \& Cardozo, S. (1995). Prevalência de tabagismo e fatores associados em área metropolitana da região Sul do Brasil. Revista de Saúde Pública, 29(1), 46-51. https://.org/10.1590/S0034-89101995000100008

Nardi, J. B. (1985). A história do fumo brasileiro. ABIFUMO, 40p. 
Research, Society and Development, v. 10, n. 3, e11210312975, 2021

(CC BY 4.0) | ISSN 2525-3409 | DOI: http://dx.doi.org/10.33448/rsd-v10i3.12975

Nogueira, A. S. (2014). Efeitos na saúde do uso de cigarros eletrónicos. Revista Portuguesa de Medicina Geral e Familiar, 30(4), 271-273.

Oliveira, W. J. C. de, et al (2018). Conhecimento e uso do cigarro eletrônico entre estudantes da Universidade Federal de Mato Grosso. Jornal Brasileiro de Pneumologia, 44(5), 367-369. Epub July 19, 2018. https://.org/10.1590/s1806-37562017000000229

Pereira A. S., et al. (2018). Metodologia da pesquisa científica. UFSM. https://repositorio.ufsm.br/bitstream/handle/1/15824/Lic_Computacao_MetodologiaPesquisa-Cientifica.pdf?sequence=1.

Pinto, M. et al(2015). Estimativa da carga do tabagismo no Brasil: mortalidade, morbidade e custos. Cadernos de Saúde Pública, 31(6), 1283-1297. Epub June 00, 2015. https://.org/10.1590/0102-311X00192013

Santos, U. P. (2018). Cigarro eletrônico - repaginação e renovação da indústria do tabagismo. Jornal Brasileiro de Pneumologia, 44(5), 345-346. https://.org/10.1590/s1806-37562018000050003

Silva, M. L., Silva, M. L., Silva, A. C. S. P., Freitas, Y. J. F. de, Borges, N. M. P., Cruz, M. C. A., Mori, A. S., Macedo, R. M., Garcia, T. R., \& Arruda, J. T. (2020). Condições que interferem na qualidade de vida do estudante de Medicina. Research, Society and Development, 9(11), e2469119640. 10.33448/rsdv9i11.9640.

Soares, T. A. B. (2018). Cigarro e outras formas de tabaco: investigação de conhecimento, atitudes e percepção de risco em estudantes de Medicina. Dissertação de Mestrado, Faculdade de Medicina de Ribeirão Preto, Universidade de São Paulo. Brasil. 123f.

Souza, M., Szklo, A., \& Almeida, L. M. (2018). Impact of the reduction of smoking prevalence on the trend of lung cancer mortality in Brazil. Tobacco Induced Diseases, 16(Suppl 1):A591. https://.org/10.18332/tid/84021

Urrutia-Pereira, M., Solé D., Chong Neto H. J., Badellino H., Acosta V., Castro-Almarales R. L., León M. G., Avalos M. M., Fernández C. C., Sisul-Alvariza J.C., Oliano V. J., \& Rinelli, P.N. (2019). Youth tobacco use in Latin America: What is the real extent of the problem? Allergologia et Immunopathologia, 47(4), 328-335. https://.org/10.1016/j.aller.2018.09.010.

WHO. World Health Organization. Tobacco. Genebra: WHO, 2020. https://www.who.int/news-room/fact-sheets/detail/tobacco. 